\title{
NeuroImage
}

\section{Neural mechanisms of advance preparation in task switching}

\author{
O. Gruber, ${ }^{\text {a,b,* }}$ S. Karch, ${ }^{\text {c }}$ E.K. Schlueter, ${ }^{\text {a }}$ P. Falkai, ${ }^{\text {a }}$ and T. Goschke ${ }^{\mathrm{d}}$ \\ a Department of Psychiatry, Saarland University Hospital, Homburg (Saar), Germany \\ ${ }^{\mathrm{b}}$ Max Planck Institute for Human Cognitive and Brain Sciences, Leipzig, Germany \\ ${ }^{\mathrm{c}}$ Department of Psychiatry, University of Munich, Germany \\ dDepartment of Psychology, Dresden University of Technology, Germany
}

Received 13 April 2005; revised 14 December 2005; accepted 20 December 2005

Available online 20 February 2006

\begin{abstract}
The preparation effect in task switching can be interpreted to reflect cognitive control processes during the interval between task-cue onset and the trial-stimulus onset which support the flexible and rapid configuration of response dispositions. However, it is an open issue what neural processes underlie this effect. In the present study, healthy volunteers underwent functional magnetic resonance imaging (fMRI) while performing a cued task switching paradigm, in which geometric objects had to be classified according to either color or shape. By manipulating the duration of the cue-target-interval (CTI) in the range between 0 and $1500 \mathrm{~ms}$, we were able to dissociate brain activity changes related to the processing of either the cue or the target. A network of frontal and parietal brain areas was activated during advance preparation for the upcoming task independent of whether the task was switched or repeated. The same brain regions also showed increased neural activity in response to targets without advance preparation in contrast to targets with advance preparation which only elicited activations in areas involved in visual processing and motor execution. These findings strongly argue for a 'task-set activation perspective' on advance preparation in task switching [Altmann, E.M., 2004. Advance preparation in task switching: what work is being done? Psychol. Sci. 15, 616-622.], whereas no empirical support could be found for the 'mental gear changing model' of task switching as no significant brain activity changes were observable in association with task switches, switch costs, or the interaction effect of advance preparation on switch costs. Finally, in the light of previous behavioral studies on interference effects of articulatory suppression on task preparation in humans, the present findings are compatible with the assumption that verbalization mechanisms, e.g., the retrieval of a verbal task or goal representation into working memory may be a functional component of advance configuration of task-sets.

(c) 2006 Elsevier Inc. All rights reserved.
\end{abstract}

\footnotetext{
* Corresponding author. Cognitive Neuroscience Unit, Department of Psychiatry and Psychotherapy, Saarland University Hospital, POB, D-66421 Homburg (Saar), Germany. Fax: +49 68411624270. E-mail address: oliver.gruber@uniklinikum-saarland.de (O. Gruber). Available online on ScienceDirect (www.sciencedirect.com).
}

Introduction

A central aspect of cognitive control is the ability to prepare for specific task requirements before one actually has to respond to an imperative stimulus. Task preparation supports flexible and rapid reactions to environmental events and enables one to plan actions in advance to environmental conditions. Specifically in humans, advance preparation may also involve verbal mechanisms due to the unique endowment of humans with language (e.g., Deacon, 1997), which may act as endogenous control processes.

The cued task switching paradigm offers a valuable method for investigating task preparation. In this paradigm, subjects are required to rapidly switch between two or more choice reactiontime tasks. The task cue indicates the task rule before the task actually has to be executed, which makes it possible to temporally dissociate task preparation from task execution. Because the task rules change between the tasks, there is a constant need for the subjects to adjust to the currently relevant task-set (Meiran, 1996; Mayr and Kliegl, 2000). This involves a cognitive control effort that according to some authors (e.g., Meiran et al., 2000) is reflected in the so-called switch costs. Consistent with this assumption that reconfiguration of a task-set takes time and requires cognitive resources, several studies using different task-switching paradigms have shown that the response time costs incurred by a switch between different tasks is substantially reduced if participants are given time to prepare for the new task prior to the imperative stimulus (e.g., Rogers and Monsell, 1995; Meiran, 1996; Meiran et al., 2000). However, it is still a matter of controversy whether a reduction of switch costs after a long preparation interval reflects the advanced preparatory reconfiguration of the task-set (Rogers and Monsell, 1995; Meiran et al., 2000), a strengthening of the task representation to overcome task-set inertia (Allport et al., 1994; Meiran et al., 2000), or a reduced proportion of trials on which participants fail to engage in preparatory control processes (De Jong, 2000). Furthermore, it has also been suggested by other researchers that the reduction of switch costs may not involve any switch-specific control process at all (Logan and Bundesen, 2003; Altmann, 2004). Recently, an alternative view on cognitive control in task switching has been put forward by Altmann (2004) which 
considers task activation (instead of task switching) to be the functional process in cognitive control. In contrast to the 'mental gear changing perspective' taken by most of the authors cited above, this 'task-set activation perspective' considers the main effect of task preparation, i.e., the overall reduction of reaction times both in switch and in repeat trials, to be an index of functional control processes whereas switch costs may emerge as a side effect, for instance, of priming (Altmann, 2004). These two models, the 'mental gear changing model' and the 'activation model', make different predictions also with regard to brain activation underlying cognitive control processes in a task-switching experiment.

To shed additional light on these conflicting hypotheses that were mainly derived from behavioral studies some neuroscientists have recently tried to dissociate the neuronal mechanisms underlying advance preparation (MacDonald et al., 2000; Sohn et al., 2000; Luks et al., 2002; Rushworth et al., 2002; Brass and von Cramon, 2002, 2004; Periáñez et al., 2004; Forstmann et al., 2005). While some authors focused on preparation for task switches (Sohn et al., 2000; Rushworth et al., 2002; Periáñez et al., 2004), task preparation was also investigated from a more general perspective (MacDonald et al., 2000; Luks et al., 2002; Brass and von Cramon, 2002, 2004). Most of these studies point to an important role of several frontal regions as key parts in a network which also involves posterior association cortices (Sohn et al., 2000; MacDonald et al., 2000; Luks et al., 2002; Brass and von Cramon, 2002, 2004; Derrfuss et al., 2004; Periáñez et al., 2004). However, within these widespread networks, different studies have also yielded substantial differences with respect to the specific brain areas activated in task switching. This heterogeneity in the empirical record may be mainly due to the use of different task switching paradigms. Correspondingly, in behavioral studies it has been shown that task preparation processes for explicit task cuing and predictability of task order might differ (Koch, 2003), and that the cue identity (whether the cue is a word or a single letter) might also involve different preparatory operations (Miyake et al., 2004).

Furthermore, task verbalization has been consistently shown to affect performance in diverse task-switching paradigms including the list paradigm or its variant, the pre-cued task sequence paradigm (Goschke, 2000; Baddeley et al., 2001; Emerson and Miyake, 2003; Kray et al., 2004; Saeki and Saito, 2004), as well as the random task cuing paradigm (Miyake et al., 2004). Based on these behavioral findings, we assumed that an important component of advance preparation may consist in the retrieval of a verbal task or goal representation into working memory (Goschke, 2000, 2003), especially if one has to switch between novel and unpracticed tasks with arbitrary s-r-mappings. Support for this verbal goal retrieval hypothesis was obtained in previous own experiments in which participants responded either repeatedly to the color or the identity of letters, or alternated between the two tasks (Goschke, 2000). When participants verbalized the next task ("letter" or "color") prior to the imperative stimulus, switch costs were reliably reduced compared to when they were given no time to prepare. Importantly, this reduction of switch costs was completely eliminated when task verbalization during the preparation interval was prevented by articulatory suppression.

The purpose of the present event-related fMRI study was to investigate brain processes associated with advance preparation in a cued task switching paradigm with a random task sequence (see also Meiran, 1996). In order to dissociate the target-related component from the preparation-related component we systematically varied the cue-target-interval (CTI) in the range between 0 and $1500 \mathrm{~ms}$. This manipulation made it possible to dissociate brain activity changes related to the processing of either the cue or the target and, consequently, to sort out the activations associated with endogenous task-management processes.

\section{Material and methods}

\section{Subjects}

12 healthy right-handed volunteers ( 7 females, 5 males) took part in our study (mean age $=25.5$ years; $\mathrm{SD}=1.7$ years; age range $=22-27$ years). They were recruited in an academic environment and were reported to be free from neurological and psychiatric disorders. Furthermore, written informed consent to participate in the study and ethical approval were obtained before the experiment.

\section{Experimental design}

Subjects underwent fMRI while performing a cued task switching paradigm, in which geometric objects differing in shape and color had to be classified according to either color or shape (Fig. 1). A task cue indicated which dimension was relevant for the response to the subsequent target in the current trial. The respective task cue was chosen pseudo-randomly for each trial and thus it was unpredictable for the subject if an upcoming trial required a task switch or not.

Stimuli were generated and presented using the ERTS software (Experimental Run Time System, Version 3.11, BeriSoft Cooperation, Frankfurt am Main, Germany). Each trial began with the onset of a cue which was presented at the center of a black screen indicating the relevant dimension for the subsequent response to

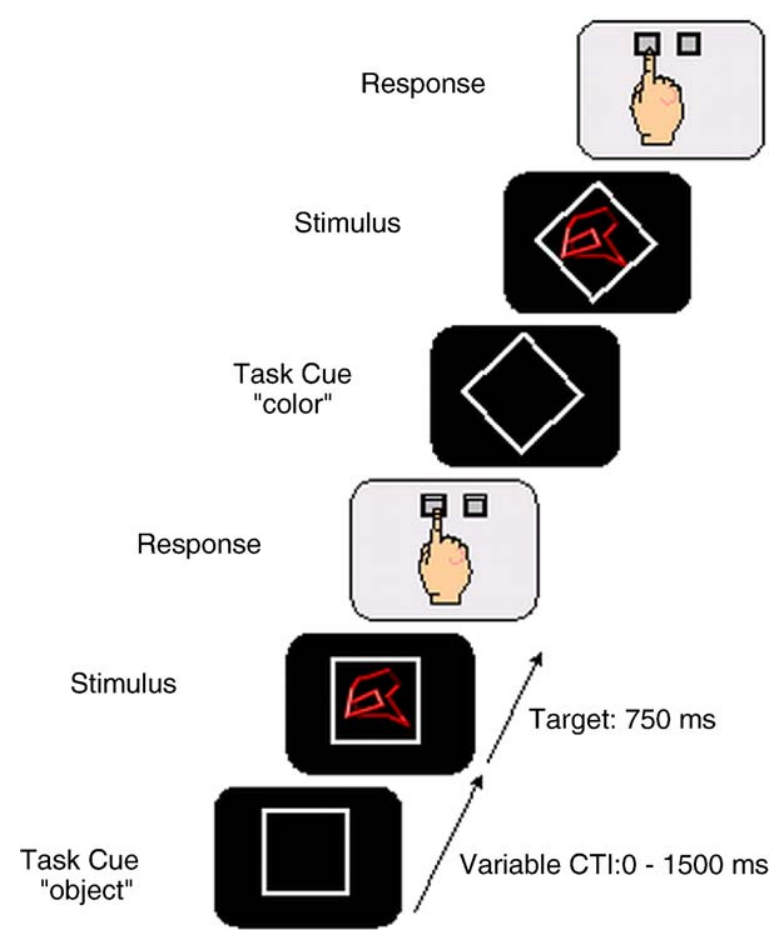

Fig. 1. Experimental design. An example of a sequence of two trials is displayed. 
the target stimulus. We used a white square as the cue for the object task and a diamond as the cue for the color task (Fig. 1). By using geometrical shapes (rather than words) as cues, we made sure that activations in language-related brain areas would not be elicited trivially due to the verbal mechanisms involved in word reading. On each trial, the cue was presented for a variable cue-target interval (CTI) which could either be $0 \mathrm{~ms}, 500 \mathrm{~ms}, 1000 \mathrm{~ms}$, or $1500 \mathrm{~ms}$. Behavioral studies typically involve comparable preparation intervals to ensure that preparation effects exert a significant influence on switch costs (Goschke, 2000; Meiran, 1996; Miyake et al., 2004). Targets consisted of two geometric figures with different shapes which were colored in red or blue with equal frequency. The target was presented on a black screen for a fixed interval $(750 \mathrm{~ms})$ and was followed by a response phase. During the presentation of the target, the cue also remained on screen and formed a frame around the target. Visual stimulation during target presentation was identical for each of the four delay-conditions, i.e., the cue always surrounded the target for the whole presentation interval.

Subjects used their right hand to respond to the target stimuli and were instructed to respond as fast and as accurately as possible. They had to press a left button with their index finger in response to the first object or the red color, and they had to press a right button with their middle finger in response to the second object or the blue color. As only one of the dimensions was responserelevant within a single trial the targets could either be congruent (i.e., both the relevant and the irrelevant dimension were mapped to the same response button) or incongruent (i.e., the two dimensions were mapped to different response buttons).

The design was completely balanced with respect to stimulus combinations, trial transitions, switch trials, trial repetitions and response congruency. In addition, every stimulus combination was equally often response-relevant or response-irrelevant. Likewise, switch trials and repeat trials occurred with identical frequency.

The trials were presented in a pseudo-random order. Altogether, subjects performed 643 experimental trials over the course of three fMRI scans. There were 6 cue-conditions and 6 target-conditions, resulting from the factorial combination of the three CTIs $(500 \mathrm{~ms} /$ $1000 \mathrm{~ms} / 1500 \mathrm{~ms}$ ) and the task-repeat vs. task-switch manipulation. Trials with a CTI of $0 \mathrm{~ms}$ on which the cue and the target were presented simultaneously, were modeled separately in the statistical analyses as they did not allow a dissociation of cue- and target-related activations.

Independently of the CTI variation, trials of each task type were also systematically jittered (with $0 \mathrm{~ms}, 375 \mathrm{~ms}, 750 \mathrm{~ms}$ or $1125 \mathrm{~ms}$ ) with respect to the onset of the cues in order to allow for oversampling of the fMRI data. The first trial of the first scan served as a dummy trial in order to assure that the design remained completely balanced as it was neither a switch nor a repeat trial and was consequently excluded from the analysis.

Subjects underwent only a short training session outside the scanner to make sure that the task was understood.

\section{fMRI measurement}

The experiment was carried out on a 3-T MRI scanner (Bruker Medspec 30/100; Bruker BioSpin MRI GmbH, Ettlingen, Germany) equipped with a circularly polarized head coil. Subjects underwent three experimental runs during each of which 604 functional image volumes were acquired consisting of twenty axial slices (voxel size $3 \times 3 \times 5 \mathrm{~mm}^{3}$, distance factor $=0.2$ ) parallel to the AC-PC plane. For this, we used a single-shot gradient EPI sequence (Inter-scan interval $1500 \mathrm{~ms}$, TE $30 \mathrm{~ms}$, flip angle $90^{\circ}$, field of view $192 \mathrm{~mm}, 64 \times 64$ matrix). In the same session, we also obtained corresponding anatomical MDEFT (modified driven equilibrium Fourier transform pulse sequence) and EPI-T1 (echoplanar imaging, t1-weighted) slices. Furthermore, in a prior session a high-resolution structural scan (3D MDEFT) was obtained for each subject.

\section{Behavioral data analysis}

Error and omission trials as well as outliers of more than two standard deviations for each trial type were excluded from the reaction time (RT) analysis. Three subjects had to be excluded from the further analysis. One was unable to complete the experiment due to claustrophobia, the other two participants committed too many errors (i.e., more than $25 \%$ ). The remaining 9 subjects performed well on the task, and individual percentages of errors did not exceed $15 \%$. Behavioral data were analyzed by means of an ANOVA with the factors duration of preparation interval (preparation effect), switch/repeat (switch costs), incongruency/congruency (response conflict), and sessions 1 to 3 (habituation effect).

\section{fMRI data analysis}

Preprocessing of the functional images used SPM99 (Wellcome Department of Cognitive Neurology, London, UK) and comprised corrections for slicetime acquisition differences, motion artifacts and low frequency fluctuations, coregistration, normalization into standard stereotactic space (using the Montreal Neurological Institute (MNI) template) and spatial smoothing with a Gaussian kernel (FWHM $=12 \mathrm{~mm})$. In event-related single-subject analyses, 6 cue-conditions and 6 target-conditions (resulting from the factorial combination of the three CTIs $(500 \mathrm{~ms} / 1000 \mathrm{~ms} / 1500$ $\mathrm{ms}$ ) and the task-repeat vs. task-switch manipulation) as well as the two (switch vs. repeat) types of trials with a CTI of $0 \mathrm{~ms}$, on which the cue and the target were presented simultaneously, were modeled as separate conditions. The resulting design matrix allowed to test for brain activity changes associated with these different events occurring at different time points in the course of the experiment. The specific statistical contrasts that were calculated are described in detail in Results. For group statistics, random effects analyses were performed on single-subject contrast images at a threshold of $P<0.001$, uncorrected. We report only those activation foci which reached a minimum cluster size of 10 voxels. For brain regions for which there was an a priori hypothesis because they had been reported to be activated during the processes under investigation in previous similar studies (e.g., Brass and von Cramon, 2002), we used small volume corrections for spheres around the previously reported activation foci in the standard Talairach coordinate system with a radius of $10 \mathrm{~mm}$. In these cases, statistical significance is reported at the level of $P<0.05$, corrected.

\section{Results}

\section{Behavioral data}

The behavioral data were analyzed with respect to both mean reaction times and error rates. Longer reaction times were 
associated with higher error rates which indicates that these effects cannot be explained simply by speed-accuracy tradeoffs.

As regards reaction times, we observed significant effects of the trial type, with switch trials producing prolonged RTs as compared to repeat trials (778 ms vs. $726 \mathrm{~ms} ; F=31.30 ; P<0.001$ ). Response incongruency of the irrelevant stimulus dimension was associated with increased RTs as well $(771 \mathrm{~ms}$ vs. $733 \mathrm{~ms} ; F=$ 12.25; $P<0.01)$. Time provided for task preparation led to a reduction of RTs (see Fig. 2; $F=95.58 ; P<0.000005$ ), whereas there was no main effect of experimental session $(F=1.91 ; P=$ 0.181 ). Furthermore, subjects exhibited a significant reduction of switch costs due to advance preparation of task switches $(F=7.38$; $P<0.005$; Fig. 2), whereas there were no habituation effects, i.e., changes of switch costs, during the course of the three experimental sessions $(F=0.14 ; P=0.867)$.

The mean error rate of the nine subjects whose functional neuroimaging data were analyzed was $6 \%$ (standard deviation $=$ $2.5 \%$ ). Mirroring the RT data, we observed a significant decrease in errors when participants had more time to prepare the task in advance (0 ms CTI: $11.8 \%, 500 \mathrm{~ms}$ CTI: 5.5\%, $1000 \mathrm{~ms}$ CTI: $3.1 \%, 1500$ ms CTI: $3.4 \% ; F=3.29 ; P<0.05)$. Incongruent trials were associated with significantly more errors than congruent trials (9.2\% vs. $2.7 \% ; F=5.70 ; P<0.05)$. Apart from that, the other effects that reached statistical significance with respect to the reaction time data (see above) did not reach the statistical criterion of $P<0.05$ when looking at subjects' error rates.

\section{Imaging data}

Analyses of the imaging data pursued three different goals: (1) the dissociation of cue- and target-related brain activity, (2) the assessment of possible neural activity associated more specifically with task switches, switch costs as well as the observed elimination of switch costs due to advance preparation, and (3) investigation of brain activity associated with the prolonged reaction times to targets without advance preparation of the task.

In the first step of analysis, we calculated the contrasts "targets versus (implicit) baseline", "cues versus (implicit) baseline" as well as the direct contrasts "cues versus targets" and "targets versus cues" in order to investigate the functional contribution of different

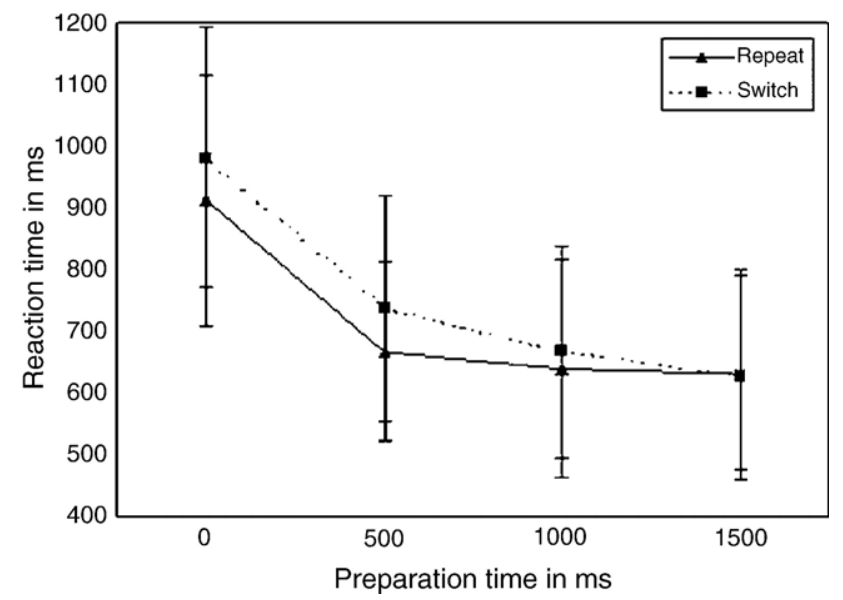

Fig. 2. Advance preparation led to reduced reaction times both in switch and repeat trials $(F=95.58 ; P<0.000005)$ as well as to a reduction of switch costs $(F=7.38 ; P<0.005)$. brain areas to cue- and/or target-related processes. Thereby, target conditions in which the cue was presented simultaneously to the target $(\mathrm{CTI}=0 \mathrm{~ms})$ were excluded because these conditions did not allow to dissociate target-related brain activations from those associated with cues. First, we investigated which brain regions were significantly activated in target conditions versus baseline. Activations associated with the target presentation and the response were found predominantly in occipital regions along the ventral visual pathway as well as in the left motor hand area, the cingulate motor area (CMA), the frontal eye field (FEF) and the cerebellum (Table 1, Fig. 3A).

Subsequently, we looked at cue-related activations. The contrast "cues versus baseline" revealed significant activations in left (and right) premotor cortices along the dorsal and posterior border of Broca's area close to the inferior frontal junction (IFJ), along the right anterior inferior frontal sulcus and middle frontal gyrus, in the left frontal eye field as well as bilaterally along the intraparietal sulcus, in extrastriate cortex and along the ventral visual pathway (Fig. 3B; see also Table 2). Importantly, activity in these brain regions was also found to be significantly enhanced in the contrast "cues versus targets" (Table 2) thus confirming the specificity of these cue-related activations as compared to brain activity related to target processing subsequent to advance preparation. Likewise, the opposite contrast, i.e., "targets versus cues", confirmed that brain activity in visual and motor areas (see above and Table 1) could be specifically attributed to the processing of targets and to task execution.

The second part of our statistical analyses of the imaging data addressed the question of whether specific neural activity can be observed in relation to task switches, switch costs and/or the elimination of switch costs that was observable after the longest preparation interval in our experiment. We looked for activations specific to task switches by comparing all cues implicating a switch with all cues implicating a task repetition, and found no significant activation related to these task differences (at $P<0.001$, uncorrected). Because switch costs were maximal without advance preparation (see Fig. 2), we assessed possible neural correlates of these switch costs by comparing brain activity during the simultaneous presentation of cue and target for task switches versus task repetitions. Again, no significant brain activity differences were found in this comparison at $P<0.001$, uncorrected. Finally, we calculated a statistical contrast aiming at the detection of brain correlates associated with the elimination of switch costs after the longest preparation interval, i.e., the interaction contrast "(target after switch and $500 \mathrm{~ms}$ CTI minus target after repeat and $500 \mathrm{~ms}$ CTI) minus (target after switch and $1500 \mathrm{~ms}$ CTI minus target after repeat and $1500 \mathrm{~ms}$ CTI)". Once again, this contrast did not show any significant brain activity changes related to the elimination of switch costs due to advance preparation. In order to substantiate the null findings reported in this paragraph, we followed the reviewers' suggestions and also used regions of interest reported in the studies by Braver et al. (2003), by Crone et al. (2005), and by DiGirolamo et al. (2001), to perform small volume corrections at $P<0.05$. Even when using this more lenient statistical criterion, we were unable to find significant activations in these ROIs related to task switches per se, to switch costs, or to the interaction effect of advance preparation on switch costs in our data set.

In the final step of the imaging data analyses, we investigated the hypothesis that similar brain processes as those underlying advance preparation for the upcoming task in general (see Fig. 3B) 
Table 1

Brain regions activated during target processing after advance task preparation $(P<0.001$, uncorrected $)$

\begin{tabular}{|c|c|c|c|c|}
\hline \multirow[t]{2}{*}{ Region } & \multicolumn{2}{|l|}{ Target vs. Baseline } & \multicolumn{2}{|l|}{ Target vs. Cue } \\
\hline & Talairach coordinates & $\begin{array}{l}\text { Statistical effects } \\
\text { ( } T \text { value })\end{array}$ & Talairach coordinates & $\begin{array}{l}\text { Statistical effects } \\
\text { ( } T \text { value) }\end{array}$ \\
\hline $\mathrm{L}$ inferior occipital gyrus & $-24-92-16$ & 8.34 & $-28-100-8$ & 4.99 \\
\hline $\mathrm{R}$ lateral occipito-temporal sulcus & $44-64-12$ & 9.10 & - & - \\
\hline L motor cortex (hand area) & $-44-3648$ & 6.62 & $-36-2464$ & 5.78 \\
\hline $\mathrm{R}$ cingulate motor area & 41636 & 5.19 & 41228 & 15.53 \\
\hline L frontal eye field & $-24-1264$ & 6.14 & $-36-2464$ & 5.78 \\
\hline L cerebellum & $-21-56-32$ & 5.83 & $-20-56-16$ & 5.35 \\
\hline $\mathrm{R}$ cerebellum & $16-56-28$ & 7.15 & $12-56-16$ & 6.50 \\
\hline
\end{tabular}

may also be observed in association with the increased reaction times to targets without advance preparation of the task. For this purpose, we compared brain responses to targets without advance preparation to neural responses to targets with advance preparation (of different durations). This contrast revealed increased brain activation to "unprepared targets" bilaterally along the dorsalposterior border of Broca's area close to the inferior frontal junction (IFJ) and in the intraparietal cortex as well as along the right anterior inferior frontal sulcus, i.e., in areas that also showed enhanced activity in association to cue presentation (see Table 2; cf. Figs. 3B and C).

\section{Discussion}

In this study we investigated neural correlates of advance preparation in task switching. For this purpose, we adopted a taskswitching paradigm that had already produced consistent results in behavioral investigations. As we wanted to separate preparationrelated from execution-related processes despite the temporal restrictions of the fMRI method we employed a manipulation of the preparation interval (ranging from 0 to $1500 \mathrm{~ms}$ ) which is commonly used in behavioral studies (Goschke, 2000; Miyake et al., 2004), but which is also applicable in an event-related fMRI

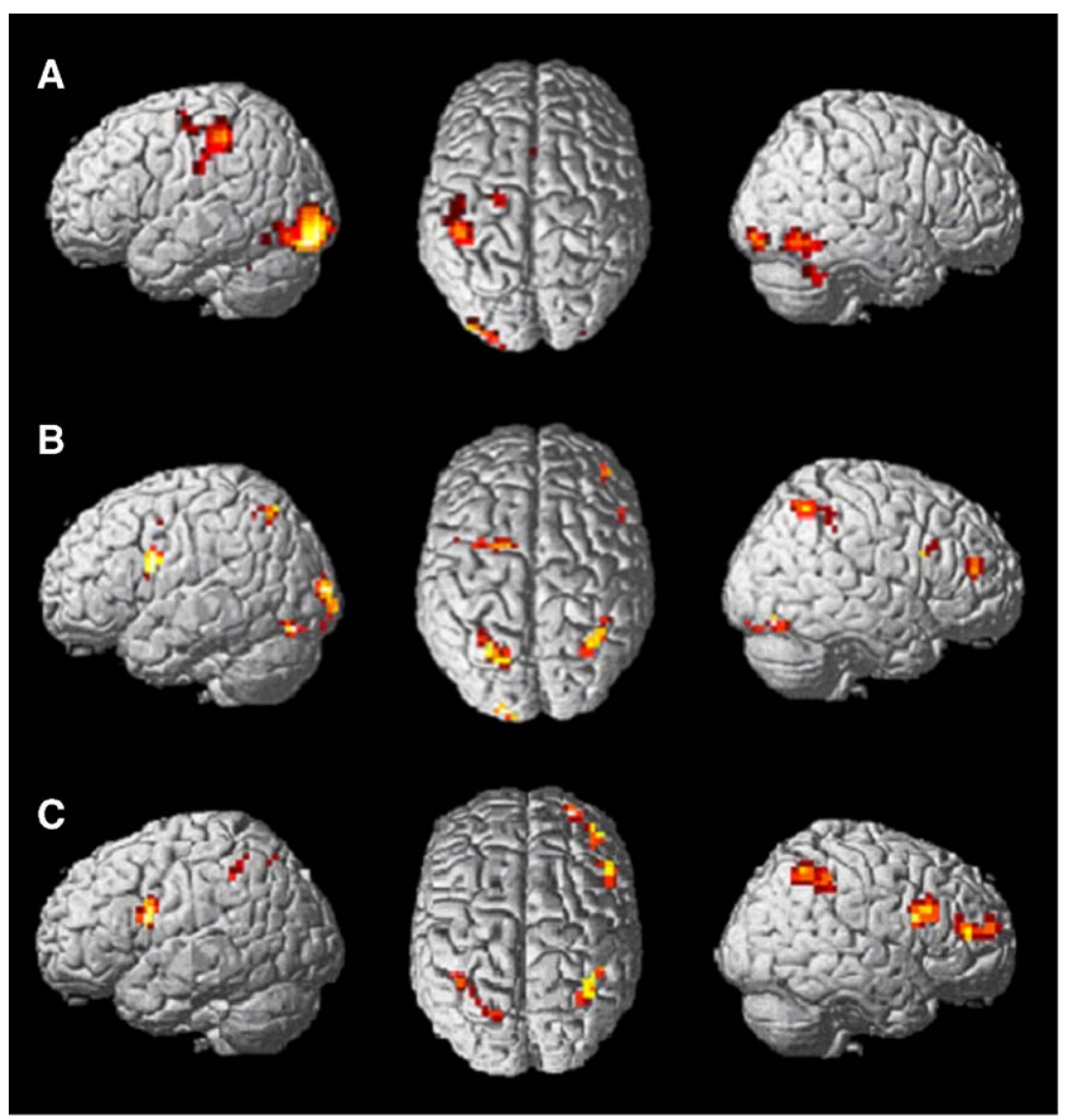

Fig. 3. Brain activations associated with (A) target processing (contrast "targets versus implicit baseline"), (B) with advance preparation in task switching (contrast "cues versus implicit baseline") and (C) with task-set activation during presentation of targets without advance preparation (contrast "targets without advance preparation versus targets with advance preparation") rendered onto a surface reconstruction of the MNI template and thresholded at $P<0.001$, uncorrected, with minimal cluster size of 10 voxels. 
Table 2

Brain regions activated during advance preparation following cue presentation and during task activation in response to targets without advance preparation ${ }^{\mathrm{a}}$

\begin{tabular}{|c|c|c|c|c|c|c|}
\hline \multirow[t]{2}{*}{ Region } & \multicolumn{2}{|l|}{ Cue vs. Target } & \multicolumn{2}{|l|}{ Cue vs. Baseline } & \multicolumn{2}{|c|}{$\begin{array}{l}\text { Targets without vs. Targets } \\
\text { with advance preparation }\end{array}$} \\
\hline & $\begin{array}{l}\text { Talairach } \\
\text { coordinates }\end{array}$ & $\begin{array}{l}\text { Statistical effects } \\
\text { ( } T \text { value })\end{array}$ & $\begin{array}{l}\text { Talairach } \\
\text { coordinates }\end{array}$ & $\begin{array}{l}\text { Statistical effects } \\
\text { ( } T \text { value) }\end{array}$ & $\begin{array}{l}\text { Talairach } \\
\text { coordinates }\end{array}$ & $\begin{array}{l}\text { Statistical effects } \\
\text { ( } T \text { value) }\end{array}$ \\
\hline L dorsal Broca's area (IFJ) & -40432 & 5.41 & -48424 & 7.82 & -52816 & 7.64 \\
\hline R dorsal Broca homologue (IFJ) & 40824 & $4.20 * *$ & 40824 & 5.15 & 441224 & 6.40 \\
\hline $\mathrm{R}$ anterior inferior frontal sulcus & 404416 & 9.03 & 444416 & 6.48 & 404016 & 6.03 \\
\hline L frontal eye field & -24052 & $4.14^{* *}$ & -24052 & 7.92 & - & - \\
\hline $\mathrm{L}$ intraparietal cortex & $-24-6040$ & 4.77 & $-24-6440$ & 11.03 & $-24-6040$ & 8.13 \\
\hline $\mathrm{R}$ intraparietal cortex & $36-5252$ & $4.50 *$ & $36-5652$ & 6.14 & $36-5252$ & 5.82 \\
\hline $\mathrm{L} / \mathrm{R}$ extrastriate cortex & $-8-100-4$ & $4.07 * *$ & $-20-968$ & 8.87 & - & - \\
\hline L lateral occipito-temporal sulcus & - & - & $-36-80-16$ & $6.21 *$ & - & - \\
\hline $\mathrm{R}$ lateral occipito-temporal sulcus & - & - & $36-76-16$ & $6.95^{*}$ & - & - \\
\hline
\end{tabular}

${ }^{\text {a }}$ All activations were significant at $P<0.05$, corrected for small volumes (with a sphere radius of $10 \mathrm{~mm}$ ), if not otherwise indicated $(* P<0.001$, uncorrected; $* * P<0.005$, uncorrected).

design. By further deploying a rather short inter-scan interval of $1.5 \mathrm{~s}$ (previous fMRI studies used an inter-scan interval of $2 \mathrm{~s}$ or more; e.g., MacDonald et al., 2000; Luks et al., 2002; Rushworth et al., 2002; Brass and von Cramon, 2002, 2004; Forstmann et al., 2005 ) in combination with systematic jittering this design achieved both a high effective temporal resolution (cf. Miezin et al., 2000) and a clear dissociation of cue- and target-related processing (see Figs. 3A and B). Thus, on the one hand, we were able to avoid long preparation intervals like the ones used in previous fMRI studies on task preparation (e.g., MacDonald et al., 2000; Sohn et al., 2000). These long preparation intervals had been criticized as to inherently involve working memory as a means for the maintenance of information leading to "trivial" activations of regions involved in verbal working memory (Brass and von Cramon, 2004). On the other hand, the experimental design of the present study also allowed to dissociate cue- from target-related processing without having to insert infrequent null events without visual stimulation as it was done in another recent study (Brass and von Cramon, 2002). These infrequent null events might entail the inherent risk to be confounded by oddball-related activations which would probably affect all subtraction-contrasts involving null events.

On the behavioral level, longer durations of the cue-target interval allowing for advance preparation led to strikingly reduced reaction times (both in switch and in repeat trials) and to a significant reduction of switch costs (Fig. 2). This finding is consistent with the results of previous behavioral studies which showed the beneficial effect of advance preparation on task performance (Rogers and Monsell, 1995; Meiran, 1996; Meiran et al., 2000). On the other hand, the observation that mean reaction times were decreased both in switch and repetition trials when increasing the preparation interval (see Fig. 2) supports the assumption that preparation processes may take place independent of whether the task is switched or repeated (see, for example, Brass and von Cramon, 2002).

\section{Dissociation of cue- and target-related brain activity}

Using the experimental variations described above, the neural processes underlying advance preparation were assessed by temporally dissociating neural activation during cue presentation from neural activation during presentation of targets after advance preparation. The corresponding statistical contrasts revealed neural responses in a fronto-parietal network (Fig. 3B, Table 2). Notably, these regions were not activated during pre-cued target processing (see Fig. 3A and Table 1), which indicates that they may represent a network specific to task preparation. When lowering the statistical criterion we further replicated a cue-related activation cluster in the pre-SMA $(x=4, y=20, z=48$; $P<0.05$; cf. Rushworth et al., 2002) that also did not occur during task execution. In striking contrast to the cue-related activity, activation related to pre-cued targets was only observed in brain areas along the ventral visual pathway subserving the processing of visual objects, on the one hand, and in regions involved in motor execution, e.g., the left primary motor cortex and the right cerebellum, on the other.

These findings are in accordance with the results of several previous studies which were interested in general aspects of advance preparation (MacDonald et al., 2000; Sohn et al., 2000; Brass and von Cramon, 2002; Luks et al., 2002) or in preparation for task switches (Sohn et al., 2000; Rushworth et al., 2002; Periáñez et al., 2004). They are also consistent with two more recent studies which tried to further disentangle processes related to simple cue encoding from endogenous control processes presumably associated with active goal retrieval and updating of the relevant task-set during task preparation (Brass and von Cramon, 2004; Forstmann et al., 2005). In these studies, the IFJ and other frontal areas as well as the intraparietal cortex and to a minor extent also the pre-SMA have been shown quite consistently to be involved in task preparation and have been suggested to reflect endogenous control mechanisms that go beyond simple cue encoding (MacDonald et al., 2000; Luks et al., 2002; Rushworth et al., 2002; Brass and von Cramon, 2002, 2004; Periáñez et al., 2004; Forstmann et al., 2005). The present study replicated this fronto-parietal network giving further support for its particular role in advance preparation in task switching. However, it is important to note that activation of these regions has been observed across different tasks addressing different processing domains and also both in switch and in repetition trials. For example, these frontal and parietal brain areas have been reported to be active during advance preparation in cued task-switching paradigms involving either semantic categorization (Sohn et al., 2000; Luks et al., 2002; Brass and von Cramon, 2002, 2004; Forstmann et al., 2005), visual categorization (Rushworth et al., 2002), or switching in a Wisconsin Card Sorting Test (Periáñez et al., 2004). Taken 
together, these studies support the assumption that the frontoparietal preparation network that was also observed in the present study is involved in the preparation of upcoming tasks and reflects general preparatory processes that are independent of paradigmspecific processing and common to both switch and repetition trials. The present study is the first to unequivocally show that these neural processes are indeed related to advance preparation, and not to task execution.

\section{Evaluating predictions of the 'mental gear changing' perspective on task switching}

Traditionally, behavioral studies have primarily focused on the effects of advance preparation on switch costs. The reason for this is, that switch costs are usually presumed to be an indicator of an endogenous control process which is characterized as "a sort of mental 'gear changing"” by some authors (e.g., Monsell, 2003). Recently however, an alternative view on cognitive control in task switching has been proposed which considers task activation (instead of task switching) to be the functional process in cognitive control (Altmann, 2004). These two models, the 'mental gear changing model' and the 'activation model', make different predictions with regard to brain activation underlying cognitive control processes in the present study. According to the 'mental gear changing model' such brain activation could be expected to be detected by statistical contrasts that, for instance, compare switch trials (that require 'mental gear changing') with repeat trials (that do not require 'mental gear changing'). By contrast, the 'activation model' would predict that the same preparatory processes are active during both task switching and task repetition, i.e., the underlying brain activation should be subtracted out in the abovementioned statistical contrast.

We addressed the predictions of the 'mental gear changing model' by searching for neural activity specifically associated with task switches (by comparing all cues implicating a switch with all cues implicating a task repetition), switch costs (by comparing brain activity during task switches versus task repetitions in trials without advance preparation) and the reduction of switch costs due to advance preparation (by calculating the interaction contrast "(target after switch and $500 \mathrm{~ms}$ CTI minus target after repeat and $500 \mathrm{~ms}$ CTI) minus (target after switch and $1500 \mathrm{~ms}$ CTI minus target after repeat and $1500 \mathrm{~ms}$ CTI)", cf. Fig. 2). None of these statistical contrasts showed any significant brain activity changes. This suggests (1) that cue-related brain activation (see Fig. 3B) was virtually identical during both task switches and task repetitions, and (2) that there were no changes of regional brain activity associated with switch costs per se and their reduction due to advance preparation. Of course, such null findings have to be interpreted very cautiously, and we cannot exclude that other studies with a higher number of subjects may reveal such activations related to task switches in general, to switch costs and/or their reduction following advance preparation. However, it is important to mention that these findings are fully compatible with recent results by Brass et al. (2003) and Crone et al. (2005). Both of these studies were equally unable to detect brain activity associated with switching processes per se, but merely reported brain activations related to task switching under bivalent response conditions only, which were correctly interpreted by the authors themselves as (probably) being related to "recoding of response meaning" and "rule representation". Furthermore, these functional neuroimaging results could be easily reconciled with the 'activation model', i.e., with the view that the same preparatory control processes operate on switch and repeat trials and that switch costs may represent an emergent property (perhaps reflecting priming effects) rather than an index of functional activity (see Altmann, 2004).

\section{Evidence for the 'task activation perspective' on advance preparation in task switching}

Another important aspect of the 'activation model' is that, rather than focusing on switch costs, it emphasizes the functional significance of the main effect of task preparation on behavioral performance which is the overall reduction of reaction times both in switch and in repeat trials. This main effect of task preparation was also the most striking behavioral effect in the present study (see Fig. 2). Therefore, we were also interested in determining the brain processes that were associated with the longer reaction times to "unprepared" targets as compared to targets presented after preparation intervals. In particular, if according to the 'activation model' the function of general preparatory control processes is to activate the current task set, one may expect the very same processes to occur in response to the presentation of targets when no time had been given to activate the current task set in advance. Indeed, when we compared targets without advance preparation to targets with advance preparation, we found enhanced brain activity in the same fronto-parietal network that was also activated during advance preparation of upcoming tasks (see Table 2; cf. Figs. 3C and B). This finding not only provides further empirical evidence for the 'task-set activation perspective' on advance preparation in task switching, but it also helps to reconcile apparently contradictory results of two previous studies.

In contrast to most prior studies on task preparation in which target-related processing seemingly was of minor interest, these two studies explicitly reported target-related brain activity (Brass and von Cramon, 2002; Luks et al., 2002). Both of these studies found several brain areas within the fronto-parietal network, whose activity was increased by cue presentation, to be also activated following target presentation. While on the first sight this might strongly argue against a role of this network in task preparation, the present results provide a clue on how to account for these findings. Luks et al. (2002), for instance, observed activation of these frontal and parietal regions only in response to neutrally cued targets, i.e., in conditions in which subjects did not get a reliable task cue in advance, but only simultaneously with the target stimulus. In this situation, which is similar to the target condition without advance preparation in our experiment, it is only possible to activate the new task by the onset of the target. In a similar way, a delayed task-set activation may also be provoked by a lack of the explicit instruction to prepare immediately after cue appearance, and this may be one possible explanation for the frontal and parietal activations observed during target processing in the study by Brass and von Cramon (2002). On the other hand, the results of the present study as depicted in Tables 1 and 2 as well as in Fig. 3 clearly demonstrate that, if subjects are explicitly instructed to prepare the tasks and if they are informed about the upcoming task in advance, the fronto-parietal network is activated exclusively in response to the cue, and not to the target. Consistent with this finding, in the study by Luks et al. (2002), those targets, which were informatively pre-cued, did not lead to an activation of these frontal and parietal areas. 


\section{Verbalization processes as a possible means for task-set activation}

In accordance with the 'activation perspective' on task switching, the present study indicates that a network of frontal and parietal brain regions is involved in the activation, i.e., configuration of task sets, either as soon as a cue allows advance preparation of the upcoming task, or at the time the target stimulus is presented together with the cue. However, the question remains which functions exactly are supported by activity in these brain regions. Cognitive psychologists have linked preparatory processes in task switching to perceptual encoding (e.g., Logan and Bundesen, 2003), memory encoding (e.g., Altmann, 2002, 2004), or to memory retrieval (e.g., Mayr and Kliegl, 2003). For instance, recent behavioral evidence suggests that the main effect of task preparation, i.e., the general reduction of reaction times in both switch and repeat conditions, is linked to the need to retain the task cue in memory (Altmann, 2004). Another line of evidence supports the assumption that task-set activation may, at least in part, rely on verbal processing. Several recent behavioral studies have shown that articulation of taskirrelevant words during the preparation interval impaired taskswitching performance and eliminated the beneficial effects of a long preparation interval, presumably due to interference with verbal self instruction (Goschke, 2000; Emerson and Miyake, 2003; Miyake et al., 2004; Saeki and Saito, 2004; see also: Mecklinger et al., 1999). While functional neuroimaging experiments certainly allow to identify neural correlates of pre-determined functional processes, they can only provide some indirect evidence to answer questions on the exact functional processes that take place in an activated brain region. Nevertheless, our finding of fronto-parietal activations related advance preparation and task-set activation, particularly in dorsal Broca's area near the left IFJ, in the left intraparietal cortex and along the right anterior inferior frontal sulcus, appears to be fully compatible with the view that verbal processes may be a component of advance configuration of task-sets because very similar brain activations have been repeatedly demonstrated to underlie the articulatory rehearsal component of verbal working memory (cf., for example, Gruber, 2001; Gruber and von Cramon, 2001; Gruber and von Cramon, 2003; Chen and Desmond, 2005). On the other hand, it is worth mentioning that the existing evidence for task verbalization to play a functional role in the advance preparation of upcoming tasks cannot be generalized to every task-switching paradigm. There certainly may be conditions in which verbal processes are not obligatory to prepare for a task, e.g., when direct cues are used, when switching is highly predictable, or when subjects have overlearned the task and have established a direct association between cues and response mapping rules. Such conditions were avoided in the present experiment. Furthermore, one may speculate that the need for (verbal) memory encoding (see Altmann, 2004) and/or (verbal) memory retrieval (see Mayr and Kliegl, 2003) may also crucially depend on the exact nature of the cue. For instance, indirect cues like the diamonds and squares used in the present study are likely to necessitate verbal memory retrieval processes in contrast to direct cues, e.g., the words "shape" and "color" (see also Miyake et al., 2004). Moreover, in particular under conditions of interference (e.g., under articulatory suppression) as well as in non-human species (e.g., see Stoet and Snyder, 2003), other, perhaps phylogenetically older neural mechanisms may account for some residual task preparation abilities. The existence of such a degeneracy of neural systems in the human brain (e.g., Tononi et al., 1999; Price and Friston, 2002) has been demonstrated in a similar way with respect to verbal working memory functions (see Gruber, 2001; Gruber and Goschke, 2004). Finally, given that verbalizing the next task has been found to facilitate performance on switch trials much more than on repeat trials (Goschke, 2000, Exp. 2; Miyake et al., 2004), one may wonder why we did not observe stronger activation in language areas on switch trials relative to repeat trials. One explanation for this finding is that in the present experiment participants had to use inner speech both on switch and repeat trials in order to recode the arbitrary task cues into a verbal task representation.

\section{Conclusions}

In the present event-related fMRI investigation of neural correlates of advance preparation in task switching, we were able to show that distinct brain areas are involved in task preparation and in the subsequent execution of the task. While advance preparation led to activation in a fronto-parietal network, processing of targets following advance preparation was merely associated with activation of brain regions subserving visual processing and motor execution. However, enhanced activity of the fronto-parietal network was also observed in response to targets without advance preparation. Consistent with the 'task-set activation' hypothesis of advance preparation in task switching (see Altmann, 2004), this result suggests that advance preparation encompasses the same processes that are also active during target presentation if no prior information was available about the upcoming task. On the other hand, we were unable to find empirical support for the 'mental gear changing model' of task switching as no significant brain activation was observable in statistical contrasts testing for neural activity associated with task switches, switch costs per se or the influence of advance preparation on switch costs. Since activation of the frontoparietal network observed in the present study has been replicated across different (e.g., semantic and visual) task switching paradigms and both in switch and in repetition trials, this network appears to represent more general preparatory processes that allow to activate the task set prior to the occurrence of the target. In the light of previous behavioral studies on interference effects of articulatory suppression on task preparation in humans, we assume that verbalization mechanisms may constitute an important means for assuring the effective configuration of the relevant task set during task preparation.

\section{Acknowledgments}

This work was supported by the German Research Foundation (DFG) in the context of the priority program "Executive Functions" (grants Gr 1950/1-1, Gr 1950/1-3, and Go 720/3-1). We thank the Max Planck Institute for Human Cognitive and Brain Sciences and in particular D. Yves von Cramon for providing the fMRI facilities that made this work possible. We also thank the reviewers of the manuscript for their very thoughtful suggestions for improvements.

\section{References}

Allport, A., Styles, E.A., Hsieh, S., 1994. Shifting intentional set: exploring the dynamic control of tasks. In: Umilta, C., Moscovitch, M. (Eds.), Attention and Performance XV. MIT Press, Cambridge, MA, pp. $421-452$. 
Altmann, E.M., 2002. Functional decay of memory for tasks. Psychol. Res. 66, 287-297.

Altmann, E.M., 2004. Advance preparation in task switching: what work is being done? Psychol. Sci. 15, 616-622.

Baddeley, A., Chincotta, D., Adlam, A., 2001. Working memory and the control of action: evidence from task switching. J. Exp. Psychol. Gen. $130,641-657$.

Brass, M., von Cramon, D.Y., 2002. The role of the frontal cortex in task preparation. Cereb. Cortex 12, 908-914.

Brass, M., von Cramon, D.Y., 2004. Decomposing components of task preparation with functional magnetic resonance imaging. J. Cogn. Neurosci. 16, 609-620.

Brass, M., Ruge, H., Meiran, N., Rubin, O., Koch, I., Zysset, S., Prinz, W., von Cramon, D.Y., 2003. When the same response has different meanings: recoding the response meaning in the lateral prefrontal cortex. NeuroImage 20, 1026-1031.

Braver, T., Reynolds, J.R., Donaldson, D.I., 2003. Neural mechanisms of transient and sustained cognitive control during task switching. Neuron 39, $713-726$.

Chen, S.H., Desmond, J.E., 2005. Cerebrocerebellar networks during articulatory rehearsal and verbal working memory tasks. NeuroImage 24, 332-338

Crone, E.A., Wendelken, C., Donohue, S.E., Bunge, S.A., 2005. Neural evidence for dissociable components of task-switching. Cereb. Cortex (electronic publication ahead of print).

Deacon, T., 1997. The symbolic species. The co-evolution of language and the human brain. The Penguin Press, London.

De Jong, R., 2000. An intention-activation account of residual switch costs. In: Monsell, S., Driver, J. (Eds.), Control of Cognitive Processes: Attention and Performance XVIII. MIT Press, Cambridge, MA, pp. 357-376.

Derrfuss, J., Brass, M., von Cramon, D.Y., 2004. Cognitive control in the posterior frontolateral cortex: evidence from common activations in task coordination, interference control, and working memory. NeuroImage $23,604-612$.

DiGirolamo, G.J., Kramer, A.F., Barad, V., Cepeda, N.J., Weissman, D.H., Milham, M.P., Wszalek, T.M., Cohen, N.J., Banich, M.T., Webb, A., Belopolsky, A.V., McAuley, E., 2001. General and taskspecific frontal lobe recruitment in older adults during executive processes: a fMRI investigation of task-switching. NeuroReport 12, $2065-2071$

Emerson, M.J., Miyake, A., 2003. The role of inner speech in task switching: a dual-task investigation. J. Mem. Lang. 48, 148-168.

Forstmann, B.U., Brass, M., Koch, I., von Cramon, D.Y., 2005. Internally generated and directly cued task sets: an investigation with fMRI. Neuropsychologia 43, 943-952.

Goschke, T., 2000. Involuntary persistence and intentional reconfiguration in task-set switching. In: Monsell, S., Driver, J. (Eds.), Attention and Performance XVIII: Control of Cognitive Processes. MIT Press, Cambridge, MA, pp. 331-355.

Goschke, T., 2003. Voluntary action and cognitive control from a cognitive neuroscience perspective. In: Maasen, S., Prinz, W., Roth, G. (Eds.), Voluntary Action. An Issue at the Interface of Nature and Culture. Oxford Univ. Press, Oxford, pp. 49-85.

Gruber, O., 2001. Effects of domain-specific interference on brain activation associated with verbal working memory task performance. Cereb. Cortex 11, 1047-1055.

Gruber, O., Goschke, T., 2004. Executive control emerging from dynamic interactions between brain systems mediating language, working memory and attentional processes. Acta Psychol. (Amst) 115, 105-121.

Gruber, O., von Cramon, D.Y., 2001. Domain-specific distribution of working memory processes along human prefrontal and parietal cortices: a functional magnetic resonance imaging study. Neurosci. Lett. 297, 29-32.

Gruber, O., von Cramon, D.Y., 2003. The functional neuroanatomy of human working memory revisited-Evidence from 3T-fMRI studies using classical domain-specific interference tasks. NeuroImage 19, 797-809.

Koch, I., 2003. The role of external cues for endogenous advance reconfiguration in task switching. Psychon. Bull. Rev. 10, 488-492.

Kray, J., Eber, J., Lindenberger, U., 2004. Age differences in executive functioning across the lifespan: the role of verbalization in task preparation. Acta Psychol. (Amst) 115, 143-165.

Logan, G.D., Bundesen, C., 2003. Clever homunculus: is there an endogenous act of control in the explicit task-cuing procedure? J. Exp. Psychol. Hum. Percept. Perform. 29, 575-599.

Luks, T.L., Simpson, G.V., Feiwell, R.J., Miller, W.L., 2002. Evidence for anterior cingulate cortex involvement in monitoring preparatory attentional set. NeuroImage 17, 792-802.

MacDonald III, A.W., Cohen, J.D., Stenger, V.A., Carter, C.S., 2000. Dissociating the role of the dorsolateral prefrontal and anterior cingulate cortex in cognitive control. Science 288, 1835-1838.

Mayr, U., Kliegl, R., 2000. Task-set switching and long-term memory retrieval. J. Exper. Psychol., Learn., Mem., Cogn. 26, 1124-1140.

Mayr, U., Kliegl, R., 2003. Differential effects of cue changes and task changes on task-set selection costs. J. Exper. Psychol., Learn., Mem., Cogn. 29, 362-372.

Mecklinger, A.D., von Cramon, D.Y., Springer, A., Matthes-von Cramon, G., 1999. Executive control functions in task switching: evidence from brain injured patients. J. Clin. Exp. Neuropsychol. 21, 606-619.

Meiran, N., 1996. Reconfiguration of processing mode prior to task performance. J. Exper. Psychol., Learn., Mem., Cogn. 22, 1423-1442.

Meiran, N., Chorev, Z., Sapir, A., 2000. Component processes in task switching. Cogn. Psychol. 41, 211-253.

Miezin, F.M., Maccotta, L., Ollinger, J.M., Petersen, S.E., Buckner, R.L., 2000. Characterizing the hemodynamic response: effects of presentation rate, sampling procedure, and the possibility of ordering brain activity based on relative timing. NeuroImage 11, 735-759.

Miyake, A., Emerson, M.J., Padilla, F., Ahn, J., 2004. Inner speech as a retrieval aid for task goals: the effects of cue type and articulatory suppression in the random task cuing paradigm. Acta Psychol. (Amst) $115,123-142$.

Periáñez, J.A., Maestú, F., Barceló, F., Fernández, A., Amo, C., Alonso, T.O., 2004. Spatiotemporal brain dynamics during preparatory set shifting: MEG evidence. NeuroImage 21, 687-695.

Price, C.J., Friston, K.J., 2002. Degeneracy and cognitive anatomy. Trends Cogn. Sci. 6, 416-421.

Rogers, R.D., Monsell, S., 1995. The cost of a predictable switch between simple cognitive tasks. J. Exp. Psychol. Gen. 124, 207-231.

Rushworth, M.F.S., Hadland, K.A., Paus, T., Sipila, P.K., 2002. Role of the human medial frontal cortex in task switching: a combined fMRI and TMS study. J. Neurophysiol. 87, 2577-2592.

Saeki, E., Saito, S., 2004. Effect of articulatory suppression on taskswitching performance: implications for models of working memory. Memory 12, 257-271.

Sohn, M.H., Ursu, S., Anderson, J.R., Stenger, V.A., Carter, C.S., 2000. The role of prefrontal cortex and posterior parietal cortex in task switching. Proc. Natl. Acad. Sci. U. S. A. 97, 13448-13453.

Stoet, G., Snyder, L.H., 2003. Task preparation in macaque monkeys (Macaca mulatta). Anim. Cogn. 6, 121-130.

Tononi, G., Sporns, O., Edelman, G.M., 1999. Measures of degeneracy and redundancy in biological networks. Proc. Natl. Acad. Sci. U. S. A. 96, $3257-3262$. 